\title{
Alpha Centauri System and Meteorites Origin
}

\author{
Gustavo V. López¹, Juan A. Nieto²,3 \\ ${ }^{1}$ Departamento de Fsica, CUCEI, Universidad de Guadalajara, Guadalajara, México \\ ${ }^{2}$ Facultad de Ciencias Fsico-Matemáticas de la Universidad Autónoma de Sinaloa, Culiacán, México \\ ${ }^{3}$ Facultad de Ciencias de la Tierra y el Espacio de la Universidad Autónoma de Sinaloa, Culiacán, México \\ Email: gulopez@cencar.udg.mx, niet@uas.edu.mx, janieto1@asu.edu
}

How to cite this paper: López, G.V. and Nieto, J.A. (2018) Alpha Centauri System and Meteorites Origin. Journal of Applied Mathematics and Physics, 6, 2370-2381. https://doi.org/10.4236/jamp.2018.611199

Received: October 22, 2018

Accepted: November 23, 2018

Published: November 26, 2018

Copyright $\odot 2018$ by authors and Scientific Research Publishing Inc. This work is licensed under the Creative Commons Attribution International License (CC BY 4.0).

http://creativecommons.org/licenses/by/4.0/

(c) (i) Open Access

\begin{abstract}
We propose a mathematical model for determining the probability of meteorite origin, impacting the earth. Our method is based on axioms similar to both the complex networks and emergent gravity. As a consequence, we are able to derive a link between complex networks and Newton's gravity law, and as a possible application of our model we discuss several aspects of the Bacubirito meteorite. In particular, we analyze the possibility that the origin of this meteorite may be alpha Centauri system. Moreover, we find that in order for the Bacubirito meteorite to come from alpha Cen and be injected into our Solar System, its velocity must be reduced one order of magnitude of its ejected scape velocity from alpha Cen. There are several ways how this could happened, for example through collision with the Oort cloud objects (located outside the boundary of our Solar System), and/or through collisions within the Solar meteorites belt (located between Mars and Jupiter). We also argue that it may be interesting to study the Bacubirito meteorite from the perspective of the recently discovered Oumuamua object.
\end{abstract}

\section{Keywords}

Complex Networks, Meteorite origin, Alpha Centauri System

\section{Introduction}

In this work we are interested in answering the question whether meteorites found on the earth actually came from the closest star Alpha Centauri (alpha Cen) system [1]. Typically when one think about a meteorite found on earth one associates its origin with a number of possibilities connected with the solar system, including asteroid belt, Kuiper belt or Oort cloud (see Refs. [1] and [2]). In a sense, this is because one assumes that the probability of the meteorite origin decreases from the sun. Thus, one determines that the most probable meteorite 
origin decreases with the distance. This way of think certainly seems to be correct but the precise mathematical argument for this view seems to be lacking. From this point of view it is intuitive to think in the alternative that the probability of meteorite origin coming from alpha Cen system is not necessarily zero. Here, we propose a mathematical model which could answer these questions. Specifically, we use the connection between complex networks [3] [4] and emergent gravity [5] proposed in Ref. [6] to develop a model for probability of meteorite origin.

Alpha Centaury system consists of three stars: the pair alpha Cen A and alpha Cen B and Proxima Cen [7]. The two main components alpha Cen A and alpha Cen $B$ are at a distance of 4.37 light-years $(1.34 \mathrm{pc})$ from the sun. Moreover, while alpha Cen A has 110 percent of the mass and 151.9 percent of the luminosity of the Sun, alpha Cen B is smaller and cooler, at 90.7 percent of the Sun's mass and 44.5 percent of its visual luminosity. During the pair's 79.91-year orbit about a common center the distance between them varies from about that between Pluto and the Sun to that between Saturn and the Sun. Proxima Cen (alpha Cen C) is at the slightly smaller distance of 1.29 parsecs or 4.24 light years from the Sun, making it the closest star to the Sun, even though it is not visible to the naked eye. The separation of Proxima from alpha Cen A and B is about 0.06 parsecs, 0.2 light years or 15,000 astronomical units ( $\mathrm{AU}$ ), equivalent to 500 times the size of Neptune's orbit. Historically, astronomers first realized that the bright star alpha Cen was a tightly orbiting pair in 1689, and Proxima Cen was first spotted in 1915 (see Ref. [1] [2] and [8] for more details).

Recently, astronomers have reported an Earth-size planet orbiting one of the nearest stars in our galaxy; alpha Cen B [9] [10]. In fact, in 2012, researchers used an instrument called the High Accuracy Radial velocity Planet Searcher to detect a planet around alpha Cen B. The instrument, which is part of the European Space Agency's La Silla Observatory in Chile, measured tiny wobbles in the star that suggested that a planet was orbiting it-likely just a bit bigger than Earth, orbiting its star every 3.24 days. Since then, researchers have tried to verify the planet's existence using transits-a slight dimming in the star as the planet passes by-but have not found additional, conclusive evidence. Previously, a number of authors used planetesimal accretion model to suggest that earth like planet can be formed in Alpha Century B (see Refs. [9] [10] and references therein). Even more recently, Proxima b, an Earth-sized exoplanet in the habitable zone of Proxima Cen, has been detected [8].

It is worth mentioning that both alpha Cen A and B are high metallicity stars, which would promote the existence of circumstellar discs with a high fraction of solid materials (meteorites) at early times (see Refs. [11] [12] [13] and [14]). Several numerical studies have shown that both stars are capable of forming terrestrial planets despite the perturbing influence of the binary companion, which appears to play a role analogous to the gas giants within our solar system. The planetesimal discs appear to be stable within $3 \mathrm{AU}$ of their parent stars, provided the inclination of the disc relative to the binary plane is less than 60 degrees (see 
Ref. [15]). However, other studies have shown that the later stage of accretion to produce lunar mass objects is reduced in efficiency due to orbital rephrasing by the binary companion. This inhibits collisional growth around alpha Cen A to regions within $0.75 \mathrm{AU}$. Moreover, visual double stars are very interesting objects for astrophysics. It is known that by determining the physical parameters of the orbits can help to determine the luminosity of such stars [16].

If circumstellar discs of alpha Cen system is capable of forming planets it is naturally to assume that there are also analogue asteroid belt, Kuiper belt or Oort cloud. From this point of view one must assume the possibility that some asteroids found on earth come from the alpha Cen system. Of course, the process can be vice versa in the sense that there has been interchange of asteroids between the alpha Cen system and the solar system. At present, roughly speaking the Oort cloud starts approximately at distance of 1 light-year and ends at 2 light-year from the sun. So one may assume that the analogue Oort cloud of the alpha Cen system starts also a distance of 1 light-year and ends at 2 light-year (from the alpha Centaury system). Considering that the average distance from the sun and alpha Centaury system is of 4 light-year it seems reasonable to assume that both Oort clouds are in interaction. If this is the case at present times presumably in the past the evolution of both systems must be of great importance in the sense that part of the matter from both systems must be interchanged. So, it is expected that some asteroids trapped in the solar system actually come from alpha Cen system. Consequently, although the meteorite origin probability found on earth is higher in the case of structure near to Earth such as the asteroid belt the probability is not necessarily zero if one assumes an alpha Cen origin of some meteorites. In this work we propose a mathematical model that could answer somehow this question. Such a model corresponds to complex network adapted to gravitational phenomena, and as a possible application of our gravitational complex network model, we consider the Bacubirito meteorite [17] [18] [19].

Finally, it is interesting to mention that a future interstellar spacecraft, including a fleet of StarChip spacecraft is currently being developed for a flyby mission by the Breakthrough Starshot project [20]. In fact, Stephen Hawking, Yuri Milner and Mark Zuckerberg helm the board for a new initiative, Breakthrough Starshot, whose technology could be used to someday reach Earth's neighboring star alpha Cen system after just a traveling at 20 percent the speed of light in a 20-year journey.

This work is organized as follows. In Section 2 we comment about complex networks. In Section 3, we obtain an expression for gravitational complex networks. In Section 4, we explore if in the case of the Bacubirito meteorite we can apply our formalism. In Section 6 we mention that the possibility that some meteorite find on earth may have interstellar origin as the Oumuamua object [20] [21] and that perhaps this may be the case for the Bacubirito meteorite. For more details of Alpha Cen System see Refs [22]-[45]. 


\section{Comments on Complex Networks}

It is known that random networks with complex topology describe a wide range of systems in Nature. Surprisingly, recent advances in this scenario show that most large networks can be described by mean-field method applied to a system with scale-free features (see Refs. [3] [4] for details). In fact, it is found that in the case of scale-free random networks, the observed power-law degree distribution is

$$
P(k) \sim \frac{1}{k^{\gamma}},
$$

where $P(k)$ is the probability that a vertex in the network is connected to $k$ other vertices and $\gamma$ is a numerical scale-free parameter so called "connectivity distribution exponent". Random networks with complex topology are based in two principles:

1) Growth: starting with small number of vertices $v_{0}$, at every time step $t$ one adds a new vertex with $e<v_{0}$ edges that will be connected to the vertices already present in the system.

2) Preferential attachment. When choosing the vertices to which the new vertex connects, one assumes that the probability $\Pi\left(k_{i}\right)$ that a new vertex will be connected to vertex $i$ depends on the connectivity (node degree) $k_{i}$ of that vertex and is given by

$$
\Pi\left(k_{i}\right)=\frac{k_{i}}{\sum_{j=1}^{v_{0}+t-1} k_{j}} .
$$

(The reacher becomes reacher.) Observe that the sum in (2) goes over all vertices in the system except the new one. Assuming that $k_{i}$ is continuous parameter, one can assume that the variation of $k_{i}$ with respect the time is proportional to this probability

$$
\frac{\partial k_{i}}{\partial t}=e \Pi\left(k_{i}\right)
$$

where $e$ is proportional constant. Thus, considering (2) we have

$$
\frac{\partial k_{i}}{\partial t}=\frac{e k_{i}}{\sum_{j=1}^{v_{0}+t-1} k_{j}} .
$$

It is possible to show that

$$
\sum_{j=1}^{v_{0}+t-1} k_{j}=2 e t .
$$

Therefore, one gets equation

$$
\frac{\partial k_{i}}{\partial t}=\frac{k_{i}}{2 t},
$$

which has the following solution (given the condition $k_{i}\left(t_{i}\right)=e$ ) 


$$
k_{i}(t)=e\left(\frac{t}{t_{i}}\right)^{1 / 2}
$$

Then, using this expression, the probability that a vertex has connectivity $k_{i}$ smaller than $k$ can be written as

$$
P\left(k_{i}(t)<k\right)=P\left(t_{i}>\frac{e^{2} t}{k^{2}}\right)=1-P\left(t_{i} \leq \frac{e^{2} t}{k^{2}}\right)=1-\frac{e^{2} t}{k^{2}\left(v_{0}+t\right)},
$$

where we have assumed that the probability density for $t_{i}$ is $P\left(t_{i}\right)=1 /\left(v_{0}+t\right)$. Making the differentiation of this expression with respect to $k$, one obtain the probability that a vertex in the network is connected to $k$ other vertices

$$
P(k)=\frac{\partial P\left(k_{i}(t)<k\right)}{\partial k}=\alpha \frac{1}{k^{3}}, \quad \text { with } \alpha=\left(\frac{2 e^{2} t}{v_{0}+t}\right) .
$$

Comparing this expression with (1), one sees that in this model the free-scaling parameter becomes $\gamma=3$.

\section{Complex Network; Gravitational Information Theory}

The idea of connecting gravity with networks has been of great interest through the years (see Ref. [5] for details). In Ref. [5] it was shown that by relaying in a connection between information theory and scale-free random networks one can obtain the Newton gravitational theory (see Appendix). In Ref. [6] the identification $P \leftrightarrow F$ of the expressions

$$
P \sim \frac{1}{k^{3}},
$$

and

$$
F \sim \frac{1}{r^{2}}
$$

was considered. Consequently the possible relation between the radio $r$ and the connectivity $k$ was established,

$$
r \sim k^{3 / 2} .
$$

In fact, the expression (10) can be generalized in the form

$$
P \sim \frac{1}{k^{\gamma}}
$$

where, as it was mentioned in section $1, \gamma$ is just a free-scale parameter called the connectivity distribution exponent. It turns out that the scale-free parameter $\gamma$ is a model dependent. For instance, in the observed networks the values in the range $2 \leq \gamma \leq 3$ [6]. For gravitational theory the most interesting possibility is when $\gamma=2$. In this case and because of the above expression, $P(k)$ becomes

$$
P \sim \frac{1}{k^{2}}
$$

and therefore one can make the identification $r \sim k$ to obtain 


$$
P \sim G \frac{M m}{r^{2}},
$$

where the constant of proportionality must have units inverse of force units. This expression can be interpreted as "the probability that object of mass $m$ is connected to other object of mass $M$ is inversely proportional to the square distance between the two masses". Thus, from of point of view of complex networks the Newton gravitational law is the emergent probabilistic expression (A7) (see Appendix), which can be used to estimate the probability for a meteorite to impact the Earth from a given location, that is, to determine meteorite origin impacting the Earth.

\section{Bacubirito Meteorite}

The Bacubirito meteorite [17] is a notorious and famous anomalous iron meteorite which was found at $25^{\circ} 42^{\prime} 05^{\prime \prime} \mathrm{N}, 107^{\circ} 54^{\prime} 19^{\prime \prime} \mathrm{W}$ in 1889 at small village called "el Camichn", about $10 \mathrm{~km}$ away from Bacubirito town located in the northern mountains of Sinaloa, México. It is worth mentioning that this location has been verified by recent expedition. At the time of its finding, it was considered the biggest worldwide meteorite. Nowadays, with its 4.1 meters it is still maintain as the world fifth largest meteorite [19].

At present, it is not known the origin of the Bacubirito meteorite. Of course, one should expect that its most probable origin is the asteroid belt, the Kuiper belt or the Oort cloud. However, since it is considered anomalous iron meteorite we would like to leave open the possibility that its origin is the alpha Cen system. According to the Ref. [13] the alpha Cen system contains high concentration of metallic substances. So if one is interested to see whether a meteorite origin is alpha Cen system one needs to look for iron anomalous meteorites which it turns out to be the case of the Bacubirito meteorite. Furthermore, just by looking the expression (15), one realize that the probability that a meteorite origin is alpha Cen system is small compared with the asteroid belt, the Kuiper belt or the Oort cloud origin because of the huge distance difference. However, it is not zero

$$
\frac{P_{\text {Alfa }}}{P_{\text {Kuiper }}}=\left(\frac{r_{K}}{r_{A}}\right)^{2} \approx 10^{-10},
$$

where $M=5.972 \times 10^{24} \mathrm{~kg}, \quad m=22 \times 10^{3} \mathrm{~kg}, \quad r_{A}=4.13 \times 10^{16} \mathrm{~m}$, and $r_{K}=1.5 \times 10^{11} \mathrm{~m}$. Moreover, alpha Cen A is one of the closest stellar systems and has spectral type very similar to the sun. There are observation results [13] that indicate that metal abundance of alpha Cen $\mathrm{A}$ is greater than the sun abundance. In fact, alpha Cen A may be classified as anomalous metal rich start. In this sense, the metal anomaly of alpha Cen A is related with the metal anomaly of the Bacubirito meteorite. But of course, to make a complete identification one needs among other studies to compare the full chemical composition of alpha Cen A and the Bacubirito meteorite.

It is known that in order to understand the evolution of the Milky Way one 
uses the chemical composition of stars. Using this data, one concludes that neighbor stars are not isolated system but rather the vicinity of one another are affected by the same astrophysical events. So it is likely that alpha Cen system and the sun interact in different forms during their evolution formation. In particular one should expect that the matter interchange between both systems was a very possible scenario. In particular, in different time periods some asteroids of alpha Cen system could reach the solar system and vice versa. In this context, the study of the comparison of chemical abundance in both neighbors stars and different meteorites found of earth can be of great importance.

We would like also to analyze the possibility that the scape velocity play an important physical role in our search for a meteorite from alpha Cen enter to the solar system. As we mentioned Alpha Cen system is mainly made up of alpha Cen A, alpha Centauri B and Proxima Centauri which is a red dwarf. They masses are respectively $m_{A}=1.1 M_{\odot}, m_{B}=0.907 M_{\odot}$, and $m_{P}=0.123 M_{\odot}$, where $M_{\odot}=1.98855 \times 10^{30} \mathrm{~kg}$. So, the total mass of the systems is $M=2.13 M_{\odot}$. Now, the scape velocity of a body associated with the system of mass $M$ is given by

$$
v=\sqrt{\frac{2 G M}{r}},
$$

where $G$ is the gravitational constant $\left(G=6.67 \times 10^{-11} \mathrm{~m}^{3} \cdot \mathrm{kg}^{-1} \cdot \mathrm{s}^{-2}\right), M$ is the mass of the system, and $r$ the center of mass of the body-system. If $r_{\odot}$ is the center of mass of a body-Solar system, the ratio between the scape velocities for both system is (total mass of our Solar System is dominated by the mass of the Sun)

$$
\frac{v}{v_{\odot}}=\sqrt{\frac{M}{M_{\odot}}} \sqrt{\frac{r_{\odot}}{r}} .
$$

Therefore, one has that this comparison ratio between alfa Cen and our Solar System is

$$
\frac{v}{v_{\odot}} \approx 1.46,
$$

where it has been assumed that $r \approx r_{\odot}$ one can guess that this object in alpha Centauri must be located between alpha Cen A and alpha Cen B where the non linear resonances can produce instabilities in the objects and can be ejected from the system, where the distance from alpha Cen A to alpha Cen B is about the distance from our Sun to Pluto, between 4.4 to 7.4 billion $\mathrm{Km}$. In this way, any object escaping from Alpha Centauri in direction to our Solar System will have a speed such that the Solar System will not be able freely captured it into its system, unless directly hit the sun of a planet; in particular the earth. Thus, a direct hit of meteorite of the size of the Bacubirito meteorite from alpha Cen will produce a crater bigger than size left by the meteorite which made the Arizona crater (about one $\mathrm{Km}$ of diameter). However, there is not such a crater on the place where Bacubirito was found. Therefore, its enter energy in Earth atmosphere 
had to be much smaller. Let us see with some details: taking $r \approx 3 \mathrm{AU}=4.5 \times 10^{8} \mathrm{~km}$ the scape speed of this object coming out of Alpha Centauri is about $v \approx 35.4 \mathrm{~km} / \mathrm{s}$ (making a trip for 37,000 years to get our Solar System, covering a distance of $41.3 \times 10^{12} \mathrm{~km}$ ). Therefore, its kinetic energy is approximately $10.5 \times 10^{12} \mathrm{~J} \approx 2.6 \times 10^{3}$ Ton (TNT) which is equivalent to one Kton atomic bomb. Therefore, in order for the Bacubirito meteorite to come from alpha Cen, its velocity must be reduced about one order of magnitude of its scape speed. There are several ways this energy can be reduced, for example through collision with the Oort sloud objects (outside the plates orbits of our Solar System) and/or meteorites belt (between Mars and Jupiter) of our Solar System.

\section{Final Remarks}

While we were preparing and refining the present article for publication we became aware of the surprisingly discovered, on 2017 October 19, of the first interstellar object called 1I/2017 U1 (Oumuamua) (see Refs. [21] and [46]). This discovered triggers the possibility that many interstellar objects has been passed through our solar system in the past [20]. Thus, one may consider the possibility that some of these interstellar objects may be reached the earth. In particular, one may assume that the Bacubirito meteorite has interstellar origin as the $\mathrm{Ou}$ muamua object. Previously, we have considered the possibility that the Bacubirito meteorite came from alpha Cen system, but the fact that the Oumuamua object has other possible origins opens other scenarios for the origin of the Bacubirito meteorite. It is worth mentioning that the surface reactivity of the $\mathrm{Ou}$ muamua object is spectrally red suggesting, among other possibilities, a surface containing minerals with nanoscale iron [47]. In fact, it is interesting to mention that the chemical composition of Bacubirito meteorite is (Fe $88.94 \%$, Ni $6.98 \%$, Co $0.21 \%$, F\%) indicating that it is very unusual with respect most of the meteorites that are assumed to come from meteorites Belt of our solar system. Thus, it will be interesting for further research to study the Bacubirito meteorite from the perspective of the Oumuamua object.

\section{Acknowledgements}

This work was partially supported by PROFAPI-UAS/2012. J. A. Nieto would like to thank J. H. Abundis, C. Añorve and E. Terán for helpful comments.

\section{Conflicts of Interest}

The authors declare no conflicts of interest regarding the publication of this paper.

\section{References}

[1] Beech, M. (2015) Alpha Centauri: Unveiling the Secrets of Our Nearest Stellar Neighbor (Series: Astronomers Universe). Springer International Publishing, Swit- 
zerland.

[2] Prialnik, D. (2000) An Introduction to the Theory of Stellar Structure and Evolution. Cambridge University Press, Cambridge.

[3] Albert, R. and Barabasi, A.L. (2002) Statistical Mechanics of Complex Networks. Reviews of Modern Physics, 74, 47. https://doi.org/10.1103/RevModPhys.74.47

[4] Barabasi, A.L., Albert, R. and Jeong, H. (1999) Mean-Field Theory for Scale-Free Random Networks. Physica A, 272, 173-187.

https://doi.org/10.1016/S0378-4371(99)00291-5

[5] Verlinde, E.P. (2011) On the Origin of Gravity and the Laws of Newton. Journal of High Energy Physics, 2011, 29.

[6] Nieto, J.A. (2013) Scale-Free Growing Networks and Gravity. Revista Mexicana de Física, 59, 201-204.

[7] Wertheimer, J.G. and Laughlin, G. (2006) Are Proxima and Alpha Centauri Gravitationally Bound? Astronomical Journal, 132, 19951997. https://doi.org/10.1086/507771

[8] Anglada-Escudé. G., et al. (2016) A Terrestrial Planet Candidate in a Temperate Orbit around Proxima Centauri. Nature, 536, 437-440. https://doi.org/10.1038/nature19106

[9] Gonzalez, A., Cardenas, R. and Hearnshaw, J. (2013) Possibilities of Life around Alpha Centauri B. Revista Cubana de Física, 30, 81-83.

[10] Milli, J., Mouillet, D., Mawet, D., Schmid, H.M., Bazzon, A., Girard, J.H., Dohlen, K. and Roelfsema, R. (2013) Prospects of Detecting the Polarimetric Signature of the Earth-Mass planet $\alpha$ Centauri B b with SPHERE/ZIMPOL. Astron. Astrophys, 556, A64. https://doi.org/10.1051/0004-6361/201321881

[11] Sheminova, V.A. and Gadun, A.S. (1998) Fourier Analysis of Fe I Lines in the Spectra of the Sun, $\alpha$ Centauri A, Procyon, Arcturus, and Canopus. Kinematika Fiz. Nebesnykh Tel, 14, 219. arXiv:1004.3286 [astro-ph.SR]

[12] Porto de Mello, G.F., Lyra, W. and Keller, G.R. (2008) The Alpha Centauri Binary System: Atmospheric Parameters and Element Abundances. Astronomy \& Astrophysics, 488, 653-666. arXiv:0804.3712 [astro-ph] https://doi.org/10.1051/0004-6361:200810031

[13] Doyle-Pegg, M.T., O’Mara, B.J., Ross, J.E. and Bessell, M.S. (2005) The Chemical Composition of $\alpha$ Cen A: Strong Lines and the ABO Theory of Collisional Line Broadening. Publications of the Astronomical Society of Australia, 22, 6-12. astro-ph/0407468 https://doi.org/10.1071/AS03054

[14] Primas, F., Duncan, D.K., Pinsonneault, M.H., Deliyannis, C.P. and Thorburn, J.A. (1997) HST Be Abundances in the Alpha Centauri System. Nuclear Physics A, 621, 23-26. https://doi.org/10.1016/S0375-9474(97)00205-4

[15] Quintana, E.V., Adams, F.C., Lissauer, J.J. and Chambers, J.E. (2007) Terrestrial Planet Formation around Individual Stars within Binary Star Systems. The Astrophysical Journal, 660, 807. https://doi.org/10.1086/512542

[16] Worth, R. and Singurdsson, S. (2016) Effects of Proxima Centauri on Planet Formation in Alpha Centauri. arXiv:1607.03090 [astro-ph.EP].

[17] Ward, H.A. (1902) On Bacubirito, the Great Meteorite of Sinaloa, Mexico. Science, 16, 395-398. https://doi.org/10.1126/science.16.401.395

[18] Terán-Bobadilla, E., Abundis-Patiño, J.H., Añorve, C., Moraila, C.R., Ortega-Gutiérrez, F. and Aragón-Calvo, M.A. (2017) On a Novel Geometric Analysis of the Bacubirito Meteorite. Earth, Moon, and Planets, 120, 101-111. 
[19] Terán-Bobadilla, E., Abundis-Patiño, J.H., Añorve-Solano, C., Moralia-Valenzuela, C.R. and Ortega-Gutierrez, F. (2018) Bacubirito: The Longest Meteorite in the World. Astronomy \& Geophysics, 59, 2.

[20] http://www.space.com/32546-interstellar-spaceflight-stephen-hawking-project-stars hot.htmlReference

[21] Meech, K.J., Weryk, R., Micheli, M., et al. (2017) A Brief Visit from a Red and Extremely Elongated Interstellar Asteroid. Nature, 552, 378-381. https://doi.org/10.1038/nature25020

[22] Hatzes, A.P. (2013) Radial Velocity Detection of Earth-Mass Planets in the Presence of Activity Noise: The Case of Alpha Centauri Bb. The Astrophysical Journal, 770, 133. arXiv:1305.4960 [astro-ph.SR] https://doi.org/10.1088/0004-637X/770/2/133

[23] Hinkel, N.R. and Kane, S.R. (2013) Implications of the Spectroscopic Abundances in $\alpha$ Centauri A and B. Monthly Notices of the Royal Astronomical Society, 432, L36. arXiv:1304.0450 [astro-ph.SR]

[24] Liseau, R., et al. (2013) $\alpha$ Centauri A in the Far Infrared: First Measurement of the Temperature Minimum of a Star Other than the Sun. Astronomy \& Astrophysics, 549, L7. arXiv:1212.3954 [astro-ph.SR] https://doi.org/10.1051/0004-6361/201220776

[25] Yildiz, M. (2011) Solar and $\alpha$ Centauri A and B Models Improved by Opacity Enhancement-A Possible Explanation for Oversize Cool Stars. Monthly Notices of the Royal Astronomical Society, 412, 2571-2578. arXiv:1101.1205 [astro-ph.SR] https://doi.org/10.1111/j.1365-2966.2010.18075.x

[26] DeWarf, L.E., Datin, K.M. and Guinan, E.F. (2010) X-Ray, FUV, and UV Observations of Alpha Centauri B: Determination of Long-Term Magnetic Activity Cycle and Rotation Period. The Astrophysical Journal, 722, 343. arXiv:1009.1652 [astro-ph.SR] https://doi.org/10.1088/0004-637X/722/1/343

[27] Siregar, S. and Kuncarayakti, H. (2007) Recalculating the Orbit of $\alpha$-Centauri AB. Jurnal Matematika dan Sains, 12, 85. arXiv:1007.2293 [astro-ph.EP]

[28] Sheminova, V.A. and Gadun, A.S. (1998) Fourier Analysis of Fe I Lines in Spectra of the Sun, Alpha Centauri A, Procyon, Arcturus and Canopus. Kinematics and Physics of Celestial Bodies, 14, 169.

[29] Xie, J.-W., Zhou, J.-L. and Ge, J. (2010) Planetesimal Accretion in Binary Systems: Could Planets Form around Alpha Centauri B? The Astrophysical Journal, 708, 1566. arXiv:1001.2614 [astro-ph.EP] https://doi.org/10.1088/0004-637X/708/2/1566

[30] Arentoft, T., Kjeldsen, H. and Bedding, T.R. (2009) Optimizing Weights for the Detection of Stellar Oscillations: Application to $\alpha$ Centauri A and B, and $\beta$ Hydri. arXiv:0901.3632 [astro-ph.SR].

[31] Thebault, P., Marzari, F. and Scholl, H. (2009) Planet Formation in the Habitable Zone of $\alpha$ Centauri B. Monthly Notices of the Royal Astronomical Society. Letters, 393, L21-L25. https://doi.org/10.1111/j.1745-3933.2008.00590.x

[32] Yildiz, M. (2008) On the Structure of the Sun and $\alpha$ Centauri A and B in the Light of Seismic and Non-Seismic Constraints. Monthly Notices of the Royal Astronomical Society, 388, 1143-1151. arXiv:0809.0015 [astro-ph] https://doi.org/10.1111/j.1365-2966.2008.13352.x

[33] Thebault, P., Marzari, F. and Scholl, H. (2008) Planet Formation in $\alpha$ Centauri a Revisited: Not So Accretion Friendly after All. Monthly Notices of the Royal Astronomical Society, 388, 1528-1536. arXiv:0806.0819 [astro-ph] https://doi.org/10.1111/j.1365-2966.2008.13536.x 
[34] Guedes, J.M., Rivera, E.J., Davis, E., Laughlin, G., Quintana, E.V. and Fischer, D.A. (2008) Formation and Detectability of Terrestrial Planets around Alpha Centauri B. The Astrophysical Journal, 679 1582. arXiv:0802.3482 [astro-ph] https://doi.org/10.1086/587799

[35] Bedding, T.R., Kjeldsen, H., Butler, R.P., McCarthy, Ch., Marcy, G.W., O’Toole, S.J., Tinney, Ch.G. and Wright, J.T. (2004) Solar-Like Oscillations in the G2 Subgiant $\beta$ Hydri from Dual-Site Observations. The Astrophysical Journal, 614, 380. astro-ph/040647

[36] Barbieri, M., Marzari, F. and Scholl, H. (2002) Formation of Terrestrial Planets in Close Binary Systems: The Case of $\alpha$ Centauri A. Astronomy \& Astrophysics, 396, 219-224. astro-ph/0209118 https://doi.org/10.1051/0004-6361:20021357

[37] Wood, B.E., Linsky, J.L., Mueller, H. and Zank, G.P. (2001) Observational Estimates for the Mass-Loss Rates of Alpha Centauri and Proxima Centauri Using HST Lyman-Alpha Spectra. The Astrophysical Journal, 547, L49. astro-ph/0011153 https://doi.org/10.1086/318888

[38] Wiegert, P.A. and Holman, M. (1997) The Stability of Planets in the Alpha Centauri System. Astronomical Journal, 113, 1445-1450. astro-ph/9609106 https://doi.org/10.1086/118360

[39] Forgan, D. (2012) Oscillations in the Habitable Zone around $\alpha$ Centauri B. Monthly Notices of the Royal Astronomical Society, 422, 1241-1249. arXiv:1202.1265 [astro-ph.EP] https://doi.org/10.1111/j.1365-2966.2012.20698.x

[40] Spiegel, D.S., Raymond, S.N., Dressing, C.D., Scharf, C.A. and Mitchell, J.L. (2010) Generalized Milankovitch Cycles and Longterm Climatic Habitability. APJ, 721, 1308-1318. https://doi.org/10.1088/0004-637X/721/2/1308

[41] Bruschi, D.E., Fuentes, I. and Louko, J. (2012) Voyage to Alpha Centauri: Entanglement Degradation of Cavity Modes Due to Motion. Physical Review D, 85, Article ID: 061701. arXiv:1105.1875 [quant-ph] https://doi.org/10.1103/PhysRevD.85.061701

[42] Liefke, C. and Schmitt, J.H.M.M. (2006) The Coronal Ne/O Abundance of $\alpha$ Centauri. Astronomy \& Astrophysics, 458, L1-L4. astro-ph/0609015 https://doi.org/10.1051/0004-6361:20066220

[43] Kervella, P., Thevenin, F., Coude du Foresto, V. and Mignard, F. (2006) Deep Imaging Survey of the Environment of $\alpha$ Centauri I. Adaptive Optics Imaging of $\alpha$ Cen B with VLT-NACO. Astronomy \& Astrophysics, 459, 669-678. astro-ph/0608510 https://doi.org/10.1051/0004-6361:20065966

[44] Kjeldsen, H., et al. (2005) Solar-Like Oscillations in Alpha Centauri B. The Astrophysical Journal, 635, 1281-1290. astro-ph/0508609 https://doi.org/10.1086/497530

[45] Miglio, A. (2004) The Effect of Using Different EOS in Modelling the $\alpha$ Centauri Binary System. AIP Conference Proceedings, 731, 187. astro-ph/0411494 https://doi.org/10.1063/1.1828404

[46] Micheli, M., Farnocchia, D., Meech, K.J., et al. (2018) Non-Gravitational Acceleration in the Trajectory of 1I/2017 U1 ('Oumuamua). Nature, 559, 223-226. https://doi.org/10.1038/s41586-018-0254-4

[47] Bailer-Jones, C.A.L., Farnocchia, D., et al. (2018) Plausible Home Stars of the Interstellar Object 'Oumuamua Found in Gaia DR2. arXiv:1809.09009v1 [astro-ph.EP] 


\section{Appendix}

\section{Gravitational information theory}

In order to explain this development let us recall how the Newton's law of gravity can be obtained from information theory (see Ref. [6]). The central idea is to assume that the space, in which one considers the motion of particles of mass $m$, is a storage of information and that this information can be storage in certain surfaces or screens. In particular one may assume that such a surface corresponds to a sphere $S^{2}$. Moreover, the information is measure by bits. Thus, one assumes that the number of bits $N$ storage in a sphere is proportional to the area $A$, that is

$$
N=\frac{A}{l_{p}^{2}}
$$

where $l_{p}$ is the Planck's length

$$
l_{p}=\sqrt{\frac{G \hbar}{c^{3}}} .
$$

In addition, one assumes the following two basic conditions for small $\Delta x$ displacement and two conditions:

1) Force $F$, entropy $S$ and temperature $T$ relation: $F \Delta x=T \Delta S$.

2) Entropy $S$ and $\Delta x$ relation: $\Delta S=\frac{2 \pi}{\lambda_{c}} \Delta x$, with $\lambda_{c}=\frac{\hbar}{m c}$ being the Compton wave length.

3) The equipartition rule for the energy: $E=\frac{1}{2} N T$, with $k_{B}=1$.

4) The rest mass equation expression: $E=M c^{2}$.

From 1) and 2) one has

$$
F=T \frac{\Delta S}{\Delta x}=\frac{4 \pi T}{\lambda_{c}}
$$

But from 3) one sees that

$$
T=\frac{2 E}{N},
$$

and using 4), one finds that $F$ becomes

$$
F=\frac{4 \pi}{\lambda_{c}} \frac{M c^{2}}{N}=\frac{4 \pi}{\hbar} \frac{M m c^{3}}{N} .
$$

This expression can also be written as

$$
F=4 \pi G \frac{M m}{N \hbar G / c^{3}}=4 \pi G \frac{M m}{N l_{p}^{2}} .
$$

Thus, using (A1) and (A2) one gets the formula

$$
F=G \frac{M m}{r^{2}}
$$

which is the familiar Newton's gravitatio law. Here, $M$ denotes the mass enclosed by a spherical screen $S^{2}$ (see also Ref. [5] for details). 